Document downloaded from:

http://hdl.handle.net/10251/135620

This paper must be cited as:

Marco, M.; Giner Maravilla, E.; Larraínzar, R.; Caeiro, JR.; Miguélez, MH. (2018). Modelling of femur fracture using finite element procedures. Engineering Fracture Mechanics. 196:157167. https://doi.org/10.1016/j.engfracmech.2018.04.024

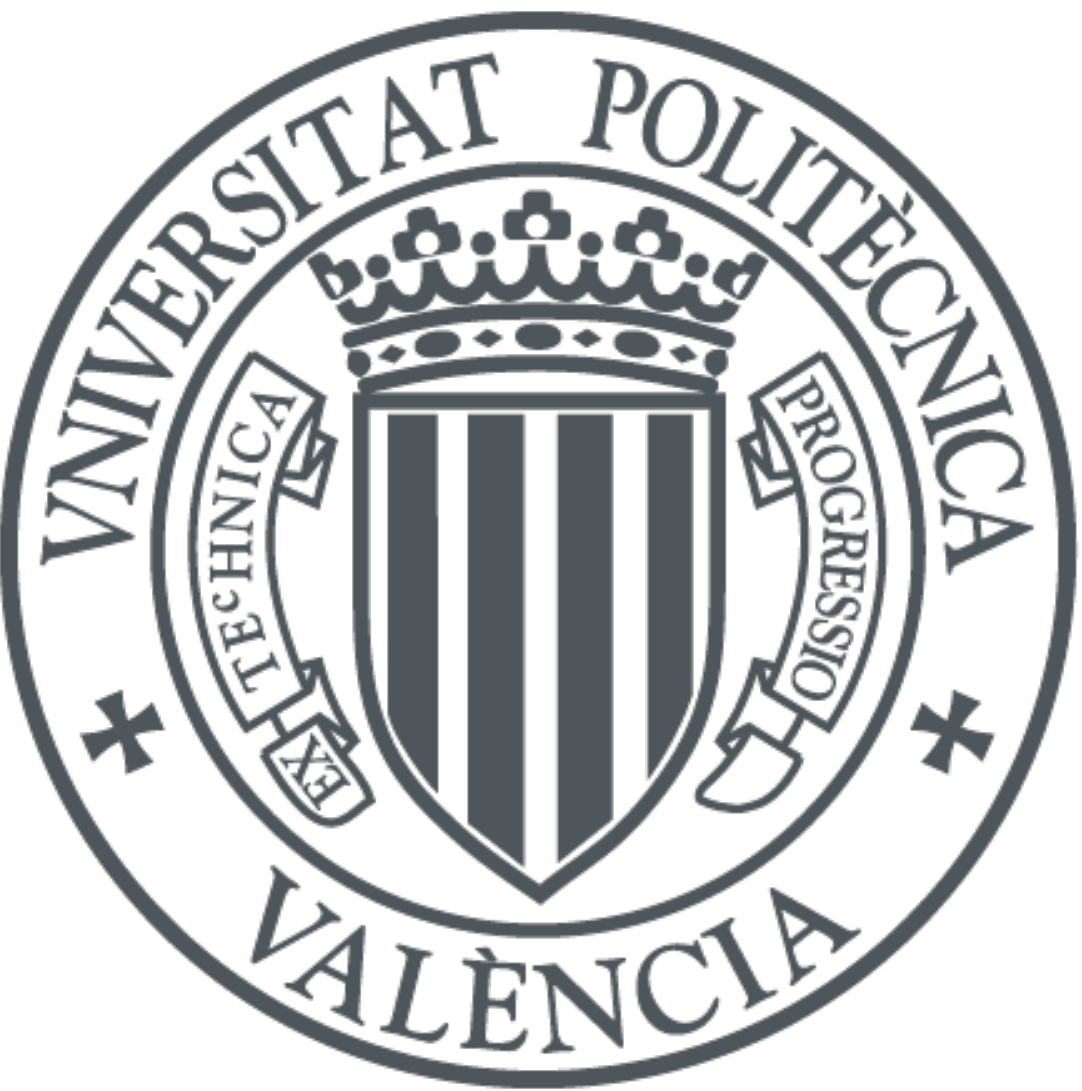

The final publication is available at

https://doi.org/10.1016/j.engfracmech.2018.04.024

Copyright Elsevier

Additional Information 


\title{
Modelling of femur fracture using finite element procedures
}

\author{
Miguel Marco $^{\mathrm{a}, *}$, Eugenio Giner ${ }^{\mathrm{b}}$, Ricardo Larraínzar-Garijo ${ }^{\mathrm{c}}$, José Ramón Caeiro ${ }^{\mathrm{d}}$, María \\ Henar Miguélez ${ }^{\mathrm{a}}$ \\ ${ }^{a}$ Department of Mechanical Engineering \\ Universidad Carlos III de Madrid. Avda de la Universidad 30, 28911, Leganés, Madrid, Spain \\ ${ }^{b}$ Center of Research in Mechanical Engineering - CIIM \\ Department of Mechanical and Materials Engineering. Universitat Politècnica de València. Camino de \\ Vera $s / n$, 46022 Valencia, Spain \\ ${ }^{c}$ Orthopedics and Trauma Department \\ Hospital Universitario Infanta Leonor. Surgery Department. Universidad Complutense Madrid. C/ Gran \\ Vía del Este, 80, 28031 Madrid, Spain \\ ${ }^{d}$ Orthopedic Surgery and Traumatology Service \\ Complejo Hospitalario Universitario de Santiago de Compostela. Rúa de Ramón Baltar, s/n, 15706 \\ Santiago de Compostela, A Coruña, Spain
}

\begin{abstract}
During the last decades human femur fracture has been mainly analysed using an experimental approach focused on cadaveric or synthetic bones. Nowadays, advances in computational technologies allow using numerical methods, such as the finite element method for femur fracture analysis. However, fracture morphology has been scarcely studied using numerical methods despite the interest of this study due to the different clinical treatment required for each fracture type. In this work, different fracture modelling techniques have been analysed with the objective of predicting a realistic fracture path, which in the literature is often limited to the initial steps of fracture. The main goal of this article is to compare different numerical approaches and to provide a robust methodology for femur fracture simulation. Experimental work was carried out on a synthetic femur in order to validate the numerical models. Through this validation we verified that some numerical methods present convergence problems, and they are not useful to model long crack paths. The best results are obtained by simulating the crack growth by a local material property degradation applied through successive analyses. This technique has been applied to a real human femur, obtaining accurate results in fracture morphology prediction.
\end{abstract}


Keywords: Femur fracture, Finite element analysis, Numerical modelling techniques, Numerical prediction of fracture

\section{List of Symbols}

$\begin{array}{cl}\rho & \text { density } \\ \nu & \text { Poisson's ratio } \\ E & \text { Young's modulus } \\ \varepsilon_{\mathrm{c}} & \text { failure strain } \\ G_{\mathrm{C}} & \text { critical energy release rate } \\ K_{\mathrm{c}} & \text { fracture toughness } \\ E_{0} & \text { initial Young's modulus } \\ \mathrm{HU}_{6} & \text { Hounsfield Units } \\ t & \text { time } \\ \rho_{\mathrm{QCT}} & \text { radiological density } \\ \rho_{\text {ash }} & \text { ash density } \\ \rho_{\text {app }} & \text { apparent density } \\ \sigma_{\text {crit }} & \text { critical stress } \\ \sigma_{\text {max,ppal }} & \text { maximum principal stress }\end{array}$

\section{Introduction}

9

\footnotetext{
${ }^{*}$ Corresponding author: Miguel Marco

Email address: mimarcoe@ing.uc3m.es (Miguel Marco)
} 
men and $14 \%$ for women in 2005 [4]. Approximately $90 \%$ of these fractures are the result of a fall [5]. Moreover diseases such as osteoporosis predispose a person to an increased risk of hip fracture [6].

The human femur has been extensively analysed through in vitro experiments in literature in order to understand its mechanical behaviour related to fracture. These experiments have provided a great knowledge of mechanical behaviour of femur, fracture loading and fracture morphology. Experimental tests evidenced that the femur behaves linearly elastic up to failure when physiological loading conditions are applied [7, 8, 9]. This idea is also corroborated by Cristofolini et al. in [10] stating that linearity holds up to the last stages of the loading path, close to the onset of fracture.

Despite the need of experiments, numerical models can also help in the understanding of femur behaviour under different load cases. In this regard, numerical models provide a useful way to understand the fracture process and, eventually, help in the assessment of fracture risk based on image diagnostics. Numerical modelling of bone fracture is a difficult task, because of the bone heterogeneity and the influence of mechanical properties of bone. It is worth noting that accurate predictions strongly depend on a realistic bone behaviour characterization. There is a wide dispersion about numerical values of bone mechanical properties in literature, due to changes in terms of age, disease, nutrition and other factors $[11,12,13]$. The dependence of the fracture load with these parameters was studied by Marco et al. in [14].

Advances in computer modelling allow the analysis of bone fracture, both at micro- and macroscale [15]. Proximal femur is the most interesting area in human femur since hip fracture commonly occurs at this zone. Linear finite element models have been successfully applied to the prediction of the elastic response and the fracture load of a human femur, with a correlation of about $90 \%$ [16]. 
The artificial, or composite femur (as usually denoted), has been commonly used in the literature as a simulant of real bone. It is important to emphasise that this kind of specimens is designed to simulate the biomechanical properties of young and healthy femurs $[17,18,19]$. These similarities were tested by means of axial compression, bending and torsion tests through the measurement of the corresponding stiffness and ultimate failure strength $[17,18]$. The use of artificial bone provides advantages for model validation avoiding the variability of properties inherent to biological tissues [18]. Composite bones are useful to develop controlled analysis, due to their homogeneous properties in two distinct zones, smoothed surface and low variability between specimens [18]. The failure modes of these composite models are close to published findings for human bones [18]. This composite femurs are useful in some clinical tasks, such as the test of a screw fixed to it [20, 21] or the behaviour of the bone after a repair through an implant or prosthesis [22, 23]. Prostheses for femur fracture have been analysed experimentally in literature joined to synthetic specimens $[24]$.

Cristofolini et al. presented a deep analysis of the synergy between experimental test and numerical models in the study of the human femur [25]. Numerical models have also been used to obtain strain values before and after a femur fracture is repaired [22, 23], and have been compared with recent measurements techniques (such as DIC, [26]) in terms of strains on the surface of the bone. These models are based on previous computed tomography (CT-scan), and they commonly analyse the stance loading of the human femur [10, 7, 27]. Using numerical methods and experimental tests has enabled to check the linear behaviour of the femur under physiological loading conditions [7] and its fracture load or global stiffness $[25,27]$.

Despite the efforts on the simulation of human femur behaviour, fracture paths have been rarely modelled using numerical approaches. Some works have focused on the fracture simulation at the proximal area, most of them obtaining small fracture paths $[14,27]$ through the XFEM method. Degradation of mechanical properties has been applied to the fracture 
modelling of human femur [28, 29], predicting more realistic and longer fracture paths.

The main goal of this work is the analysis of different approaches to model the fracture propagation in the proximal zone of the femur. These techniques are: eXtended Finite Element Method (XFEM), material property degradation at element level, element deletion and other variants with incremental crack growth. Validation was carried out using a human bone simulant (synthetic femur) because of the simplicity of this femur (composed only by two homogeneous materials representing trabecular and cortical bone) and also with application to a real human femur. The final objective of the work is to develop a technique able to model realistic fracture paths, since simulation of long fracture paths can be useful in order to predict different fracture morphologies in human femur. Once the method was validated, it has been applied to simulate other loading configurations and bone mechanical properties, including degradation of properties due to bone pathologies. There is a lack of works focused on the comparison of different numerical modelling techniques for fracture simulation in biomechanical applications. In addition, works in the literature only simulate the initial steps of fracture. It is important to establish a numerical technique able to accurately predict long fracture paths, since the further treatment strongly depends on fracture morphology.

\section{Materials and methods}

The experimental work and numerical model validation on a bone simulant is detailed in a previous work of the authors [14]. We focused on testing a synthetic bone under different loading conditions. Firstly, the femur was loaded in the elastic regime and finally the load was increased up to femur fracture. In [14], the numerical model was validated both in the elastic regime and in terms of fracture load comparing with experimental results, showing reasonably accuracy. The numerical procedure in [14] just involved the XFEM method as available in the commercial code Abaqus, being able to simulate only the onset of the fracture. The main motivation of this work is developing a numerical procedure to simulate long 
fracture paths in femur modeling and correlate the predictions with a real human femur fracture. As explained in the section Introduction, the syntethic femur presents a mechanical behaviour similar to healthy human femur [17, 18, 19]. A composite femur (fourth generation, model no. 3406, Sawbones Europe AB, Malmö, Sweden) was used in experimental analysis and modelled for numerical simulations. The artificial femur specimen is based on two different materials simulating external cortical bone (with variable thickness) and inner trabecular bone. The study of different numerical techniques is easier in a synthetic femur, since it is composed only by two homogeneous materials, unlike real human femur with an heterogeneous distribution strongly dependent on the individual age, gender and potential diseases.

\subsection{Experimental test for model validation}

Model validation (both in elastic regime and fracture load) was carried out testing the proximal femur model in a $100 \mathrm{kN}$ universal hydraulic testing machine (INSTRON 8801, load cell $100 \mathrm{kN}$ ) [14]. Three different values of load were applied on the femoral head (250 $\mathrm{N}, 500 \mathrm{~N}$ and $750 \mathrm{~N}$ ensuring that the femur was loaded in an elastic regime). The stance loading configuration was simulated (also considered by Cristofolini et al. as involves the highest risk for fracture occurrence [10]). In this loading condition, the femur was aligned by rotating the long axis of the femur to $8^{\circ}$ adduction in the frontal plane by means of an appropriate rig support. Strains on the surface of the bone were registered for each load using uniaxial strain gauges (4 in the diaphysis and 4 in the femoral neck) adhered to the femur surface. The experimental setup and the testing rig are shown in Fig.1(a) and Fig.1(b) respectively.

The numerical model of the proximal femur was developed in Abaqus/Standard. The geometry of the specimen was acquired using a CT-scanner (SIEMENS Somaton) with a pixel size of $0.44 \mathrm{~mm}$ and a slice thickness of $1.0 \mathrm{~mm}$. The image treatment, the bone modelling and the numerical model meshing were carried out through the software ScanIP 
(Simpleware, Exeter, UK). Details of the segmentation and the development of the numerical model can be found in a previous work [14].

Strains measured during experiments were compared with those predicted with the numerical model, Fig. 1(d). A total of 24 validation points were compared (obtained from 8 strain gauges and 3 loading cases). Good correlation between experimental tests and numerical model was obtained. The average relative errors between model and experimental strains were about $9 \%$, being a reasonable value when compared with other results in the literature (see for instance [27]). Concerning the fracture load of the specimen, a maximum value equal to $6330 \mathrm{~N}$ was obtained from the experimental test, while the numerical model predicted a value equal to 6069 N, with a relative error of 4\%. Fracture load was assumed equal to the value causing a node to reach the critical strain of the synthetic cortical bone. The fracture path obtained in this experimental test under stance loading is shown in Fig. $1(\mathrm{c})$.

Once the numerical model was validated in elastic regime and fracture load, different numerical techniques have been used in order to establish the most useful methodology to simulate femur fracture crack growth.

\subsection{Numerical modelling approaches}

Different numerical methodologies are analysed in this section to model fracture evolution in the femur. All the techniques studied are based on the same numerical model and only the method for fracture modelling was varied. The aim is obtaining long and realistic fracture paths, avoiding convergence problems in the numerical model.

A sensitivity analysis of the mesh was carried out in order to select a proper element size. The global stiffness of the femur was analysed versus different element sizes and finally the mesh chosen was the one for which the variation in the estimated stiffness was negligible 


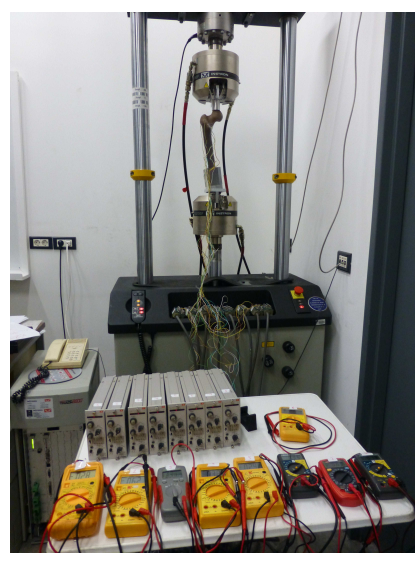

(a) Experimental setup for femur test.

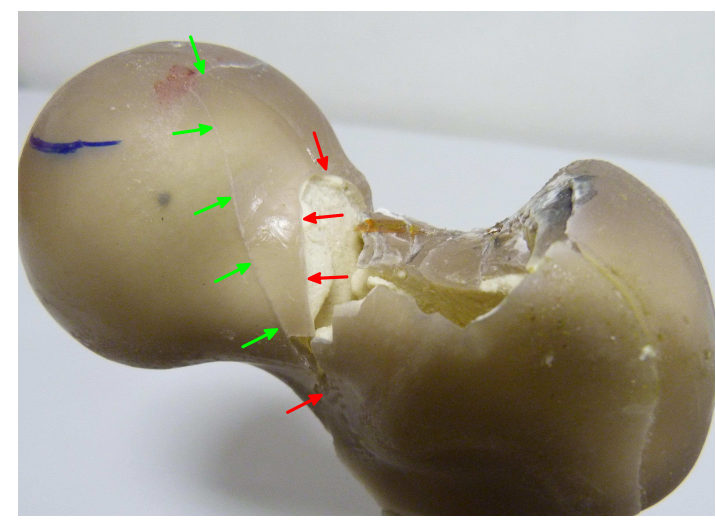

(c) Fracture obtained experimentally under

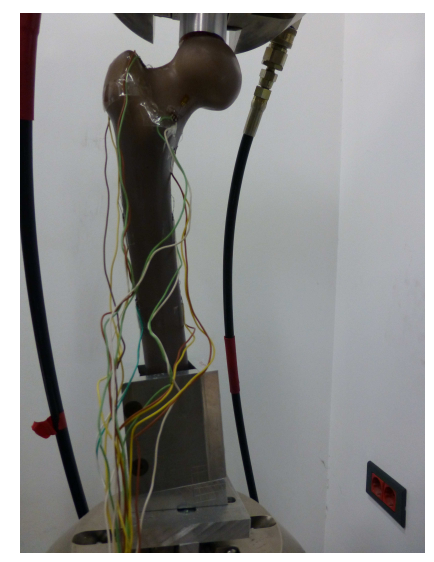

(b) Detail of the experimental rig.

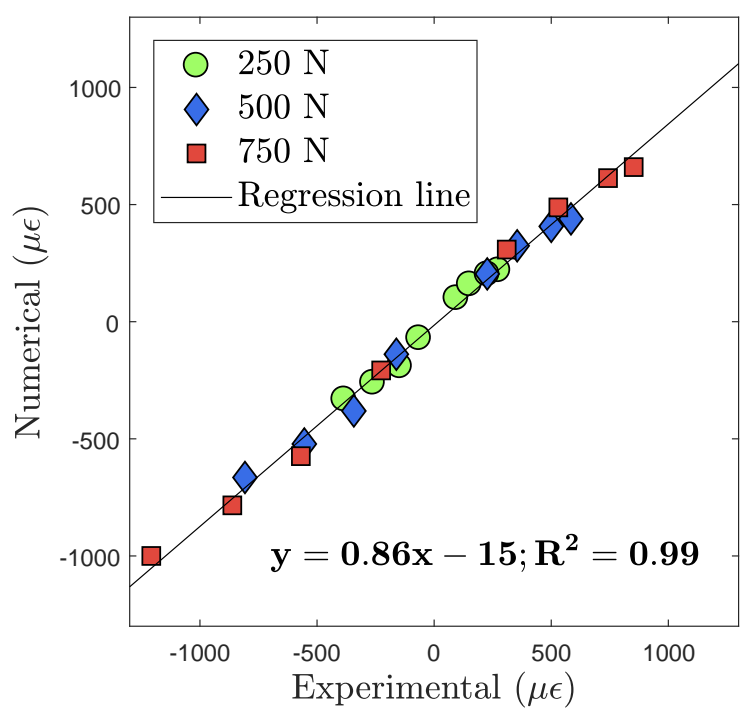

(d) Stiffness validation: comparison between exstance loading conditions. Arrows in different perimental and numerical strains. color show the two principal fracture paths obtained.

Figure 1: Validation developed in a synthetic femur in a previous work by the authors [14]. 


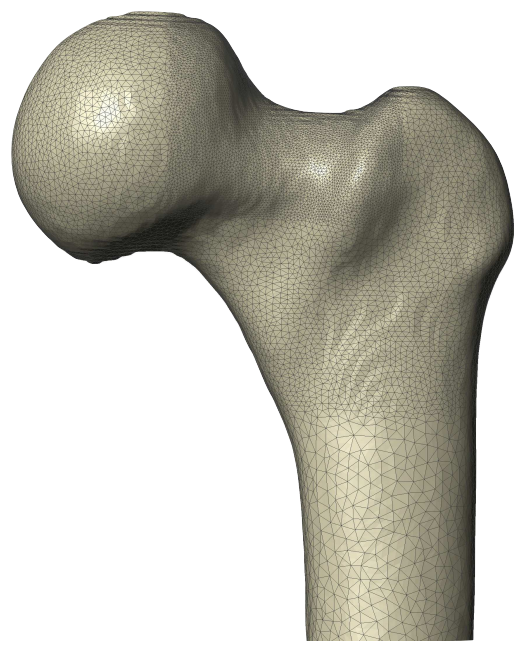

(a) Femur mesh showing the refined area at femoral neck.

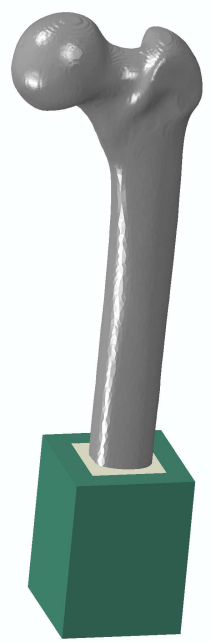

(b) Numerical model including the rig used in the experiments.

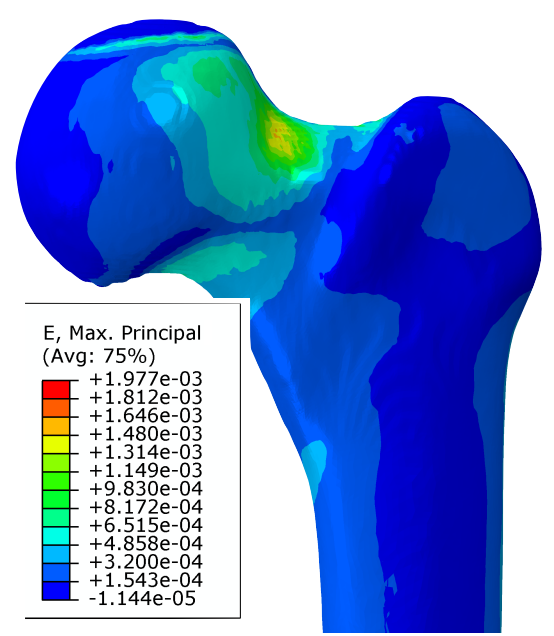

(c) Maximum principal strains in femur, under stance loading (750 N), numerical model developed and validated in previous work [14].

Figure 2: Composite femur numerical model.

tical diaphysis and then refined up to size of $2 \mathrm{~mm}$ in the cortical proximal zone (a similar element size was reported in [27]). Fig. 2(a) shows the mesh with the refined areas, while Fig. 2(c) shows the maximum principal strains in the model under stance loading. The neck zone undergoes elevated stresses and usually experiences the onset of fracture. Therefore, the mesh is refined at this zone with an element size equal to $1 \mathrm{~mm}$ in order to achieve an accurate solution in the expected fracture area. The trabecular zone was meshed with an element size equal to $3 \mathrm{~mm}$. The femur was meshed with a total number of 184400 quadratic tetrahedral elements (type C3D10 in Abaqus) and 295922 nodes. 
different mechanical properties of the rig materials were considered in the model (the white zone corresponds to surgical cement and the green zone to aluminium). Mechanical properties of cortical and trabecular bone as considered in the model are summarized in Table 1. Values marked with an asterisk (*) were calculated experimentally in a previous work [14], while the rest were provided by the manufacturer. Mechanical properties of cortical bone ( $E$ and $\varepsilon_{\mathrm{c}}$ ) were experimentally estimated, since cortical bone has a strong influence on the fracture load [14].

Table 1: Mechanical properties used in the numerical model.

\begin{tabular}{l|cc} 
Property & Trabecular bone & Cortical bone \\
\hline Density - $\rho\left(\mathrm{g} / \mathrm{cm}^{3}\right)$ & 0.27 & 1.64 \\
Poisson's ratio - $\nu$ & 0.3 & 0.3 \\
Young's modulus - E $(\mathrm{MPa})$ & 155 & $10400^{*}$ \\
Failure strain - $\varepsilon_{\mathrm{c}}$ & 0.0387 & $0.0165^{*}$
\end{tabular}

Once the different aspects of the numerical model and its main mechanical properties have been described, the different ways to model crack initiation and propagation will be explained in the following sections. In these techniques, fracture initiation, element degradation or element deletion were applied when critical strains were reached $\left(\varepsilon_{\mathrm{c}, \text { trab }}=0.0387\right.$ and $\left.\varepsilon_{\mathrm{c}, \mathrm{cort}}=0.0165\right)$. In these models it is necessary to consider a large displacement formulation due to the presence of fracture.

\subsection{1. eXtended Finite Element Method (XFEM)}

The XFEM method [30] enables the introduction of crack surfaces that are independent of the mesh geometry (they do not need to conform to element sides) which is a great advantage for crack modelling using the finite element method. This task is carried out by means of an enrichment of the elements. Thus, additional degrees of freedom are added to the nodes belonging to enriched elements. This way, the model is able to capture the discontinuity that 
fracture induces. The crack onset was predicted through the initiation criterion based on the maximum principal strain. The propagation was simulated using the XFEM capability available in Abaqus/Standard, using the Virtual Crack Closure Technique (VCCT) with mixed mode behaviour based on the Benzeggagh-Kenane expression. Regarding the critical energy values $\left(G_{\mathrm{c}}\right)$, necessary to predict the onset of crack growth, they have been estimated from the fracture toughness $K_{\mathrm{c}}$, which is related to human bone density through Eq. 1, given in [31]. Although this equation was proposed for trabecular bone, it has been successfully used in similar works where human femur fracture has been analysed [27]. The following expressions determine these relationships:

$$
\begin{gathered}
K\left(\mathrm{Nm}^{-1.5}\right)=0.7413 \cdot 10^{6} \cdot \rho^{1.49} \\
G\left(\mathrm{Jm}^{-2}\right)=\frac{K^{2}\left(1-\nu^{2}\right)}{E}
\end{gathered}
$$

where plane strain has been assumed and the ratio between different fracture modes in terms of $G_{\mathrm{c}}$ for human bone was proposed by [32]:

$$
G_{\mathrm{IIC}} / G_{\mathrm{IC}}=G_{\mathrm{IIIC}} / G_{\mathrm{IC}}=0.33
$$

Eq. 3 was calculated in [32] for cortical bone when crack orientation is orthogonal to osteons. In this work we extend this expression for any orientation of the crack. The same relationship has been used in other works for modelling human femur fracture [27].

\subsubsection{Mechanical properties degradation through USDFLD subroutine}

This technique is based on the reduction of the Young's modulus of the damaged elements up to a very low value $(E=1 \mathrm{MPa})$ to simulate the loss of stiffness due to the crack growth. An option for property degradation is available in Abaqus/Standard through a user subroutine, this method is similar to the element deletion technique. However, element deletion is not recommended in standard analysis because it leads to convergence problems. A USDFLD subroutine has been developed in order to apply the degradation of elastic 
properties. By means of this subroutine, the Young's modulus of the material in an element is decreased, depending on the value of its maximum principal strain. The maximum principal strain governs the crack propagation through the parameter $f$, according to the expression:

$$
f=\varepsilon_{\text {max }, \text { ppal }} / \varepsilon_{\mathrm{c}}
$$

where $\varepsilon_{\text {max,ppal }}$ is the maximum principal strain evaluated at each element and $\varepsilon_{\mathrm{c}}$ are the critical strains given above (distinguishing between cortical and trabecular bone). According to this parameter, mechanical properties of the elements are degraded when $f=1.0$. The following techniques are also based on this parameter. In this case, two different methods have been applied to the numerical model: first, a mechanical property reduction of $10 \%$ of their initial values, and secondly, a progressive reduction of 50\%-10\%-1\%, step by step.

\subsubsection{Element deletion through VUSDFLD subroutine}

Given that element deletion is not recommended in standard analysis, an explicit analysis has also been carried out. In this case, a VUSDFLD subroutine has been developed in Abaqus/Explicit to remove elements of the model that reach the critical strain. Similarly to the previous technique, the user subroutine compares the maximum principal strains with critical strains of each material. According to this parameter, elements are deleted when $f=1.0$.

\subsubsection{Element deletion through incremental crack growth}

Previous methods explained above in this work showed convergence problems, due to the instability introduced by the crack in the numerical model. Due to this fact, it is difficult to obtain long fracture paths required to analyse the fracture morphology. Automatized successive analyses were developed in order to improve the crack simulation. Similar techniques have been applied in other fracture problems (see for instance [33]) in order to obtain long crack paths. This method has been carried out through a Python script that interacts with 


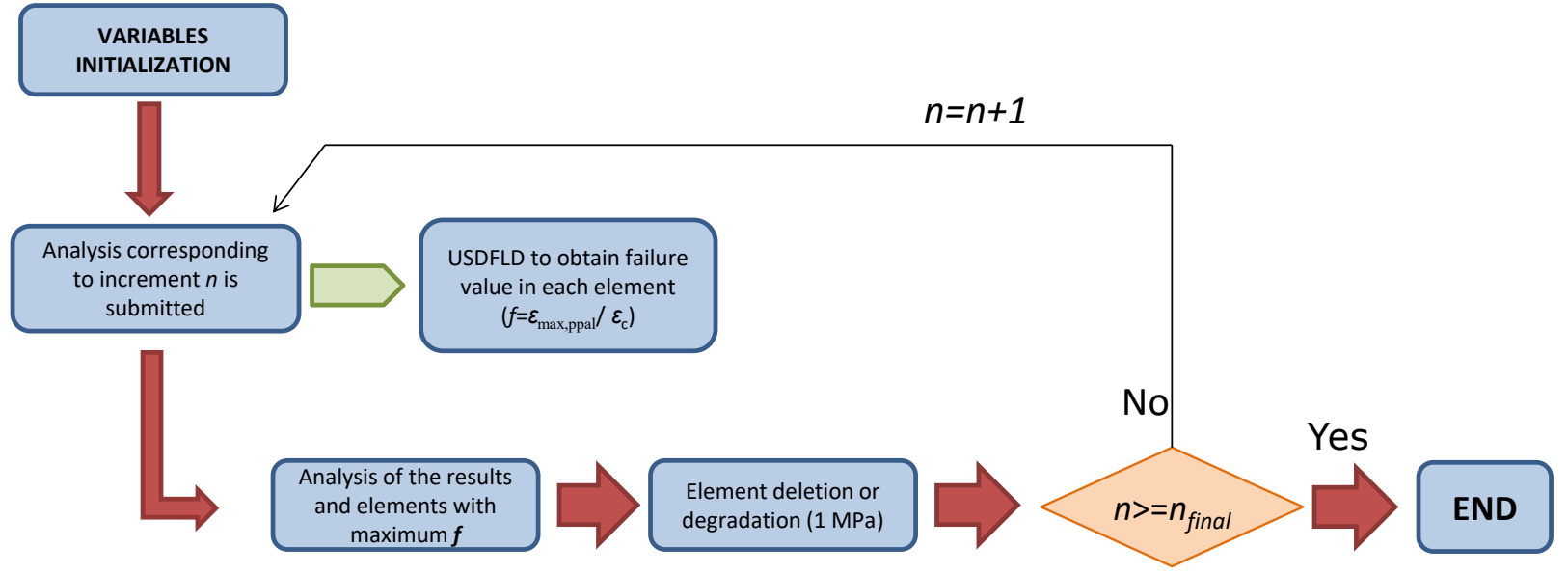

Figure 3: Scheme of the successive analysis programmed through a Python script.

Abaqus. Using this technique, each crack increment is considered as a new analysis an thus it is possible to simulate long fracture paths without convergence problems. The scheme of the incremental crack growth method is shown in Fig. 3. The maximum principal strain governs the crack modelling, and therefore elements with maximum values of $f$ are deleted.

\subsubsection{Mechanical properties degradation through incremental crack growth}

This technique is similar to that described in the previous subsection. The main difference is that elements are not deleted in the model; only their Young's modulus is degraded up to minimal values $(E=1 \mathrm{MPa})$ in order to reduce the element stiffness up to negligible values. This technique improves the distortion problems that appear when elements are deleted. Thus, the elements are preserved in the model with negligible stiffness. The scheme of the automatized process is also illustrated in Fig. 3.

\section{Results and discussions}

\subsection{Stance loading conditions}

The results obtained with each modelling technique are presented in this section. Firstly, the results corresponding to stance loading (for adduction equal to $8^{\circ}$ in the frontal plane) 


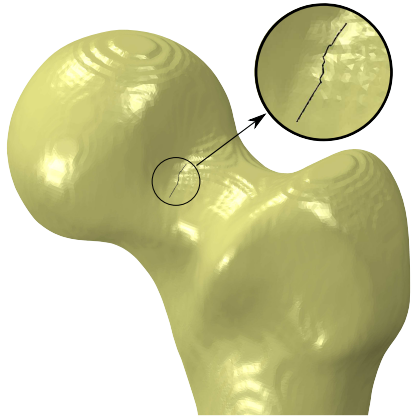

(a) XFEM as available in Abaqus/Standard v6.12

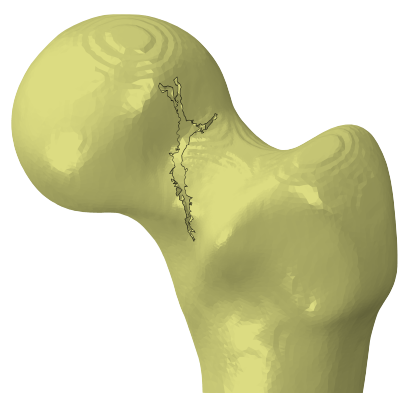

(d) Element deletion through VUSDFLD

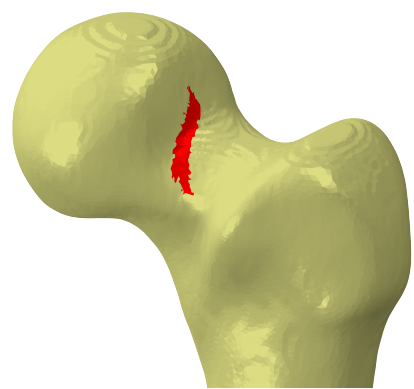

(b) Mechanical property degradation through $(10 \%)$

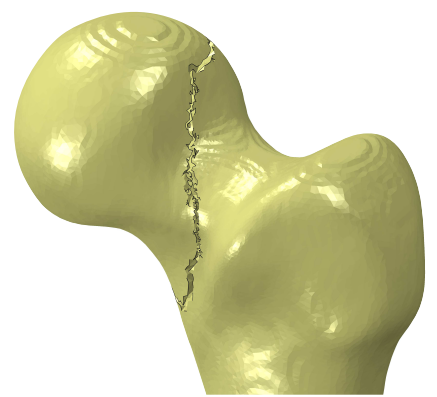

(e) Element deletion through incremental crack growth

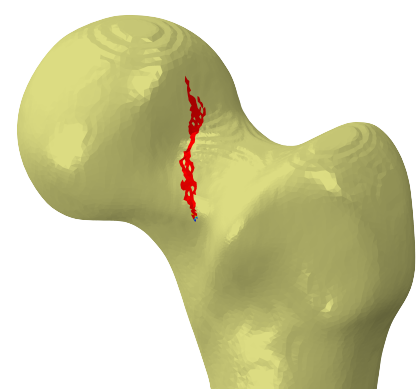

(c) Mechanical property degradthrough USDFLD (50\%$10 \%-1 \%$ )

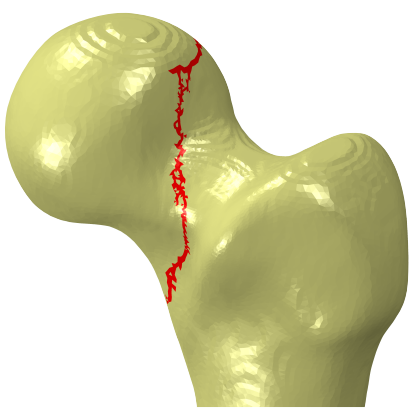

(f) Mechanical property degradation through incremental crack growth

Figure 4: Different crack propagation methods analysed in this work in the composite femur.

the crack growth for all the numerical techniques considered. The predicted fracture paths are in good agreement with experimental results. Experimental fracture showed two different paths (marked in green and red color in Fig. 1(c)), although only one of them grew enough leading to complete fracture (marked in red color). The other crack did not progress, pre- 
sumably due to the presence of the load application system on the femoral head. Fracture paths obtained in our numerical models are very similar to this latter path marked in green color in Fig. 1(c).

Fig. 4 shows that the techniques based on incremental crack growth lead to longer fracture paths than the XFEM method as implemented in Abaqus/Standard v6.12, due to convergence problems. Degradation through USDFLD and VUSDFLD leads to longer paths than that obtained with XFEM, but not enough to properly reproduce the fracture morphology. Small differences are found between degradation 50\% and degradation $50 \%-10 \%-1 \%$, although the latter presented a thinner crack path. All these methods showed poor results in terms of long crack paths, due to convergence problems.

As a result, paths obtained through incremental crack growth presented good behaviour concerning convergence, thus leading to long paths. Convergence problems are avoided with this process, because each increment of the fracture growth is a new simulation. Comparing both techniques, element deletion presents more problems, due to the presence of distorted elements, which can slow down the numerical process. Therefore, the technique of element degradation through incremental crack growth leads to the best results, in terms of convergence and fracture path length, and it can be used in other loading configurations. Results obtained with this technique for stance loading are shown in Fig. 5.

Results in Fig. 5(a) and Figs. 5(b) and 5(c) show a close match of experimental and numerical fracture patterns. In this case, fracture crosses the femoral neck, in the side closest to the femoral head up to the final fracture of the femur.

Numerical results show an accurate prediction of the initial crack path obtained experimentally. Most authors also predicted the initial steps of fracture process, see for instance $[14,27]$, although their numerical techniques were not able to simulate long fracture paths. Only Hambli et al. [29] simulated long fracture paths in a human femur using an element 

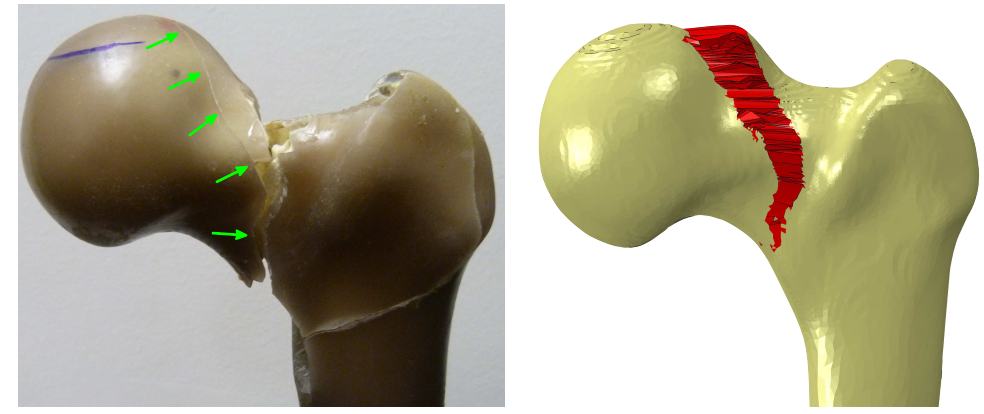

(a) Fracture obtained experi- (b) Fracture with degraded ele- (c) mentally marked in green color ments in red colour

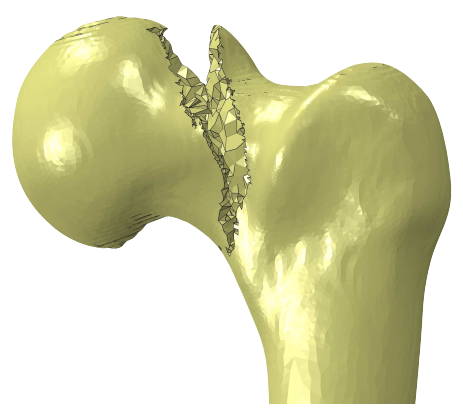

(c) Same fracture without showing degraded elements

Figure 5: Femur fracture obtained experimentally and by means of degradation of mechanical properties through incremental crack growth in stance loading.

deletion technique. With the technique proposed in this work, fracture simulation is a more controlled process, and longer fracture paths can be obtained.

Once a reliable technique has been chosen to model femur fracture, it has been used to simulate other loading and bone conditions. In this case a sideways fall conditions was simulated, and also a femur with mechanical properties corresponding to osteoporotic bone. Finally, the most reliable technique has been applied to a real human femur in order to analyze the capabilities of the technique in a real case of study.

\subsection{Other conditions (sideways fall configuration and osteoporotic bone)}

Sideways fall configurations have been commonly studied in literature, since it is estimated that $90 \%$ of these femur fractures occur as a result of a fall to the side [5]. In our simulation, the sideways fall loading condition consists of a load applied to the femoral head at $20^{\circ}$ in anteversion and $30^{\circ}$ in rotation. It was established by [8] as the most critical scenario, since it leads to the minimal fracture load in a human femur when falling conditions are studied. Results of the analysis under these loading conditions are shown in Fig. 6(a).

On the other hand, osteoporosis is a typical pathology that reduces bone strength, which 
increases the risk of a fracture, commonly at hip joint. Our numerical model able to reproduce femur fracture is used to study the osteoporotic femur behaviour. In this case, the mechanical properties corresponding to an osteoporotic bone have been implemented in the model. In this case, the femur has been analysed under stance loading conditions, the same used in previous section. It is known that osteoporosis mainly affects to trabecular bone, but, it also has an influence in cortical bone. In trabecular bone, osteoporosis increases its porosity, reducing its stiffness [34]. Regarding cortical bone, its shell becomes thinner and its porosity also increases [35] when osteoporosis is present. Hence, osteoporosis implies a reduction in stiffness, both in cortical and trabecular bone. Young's modulus of trabecular bone has been reduced following expressions proposed in [36]. Lubarda et al. proposed a numerical law that establishes the relationships between time (in years) and ratio $E / E_{0}$ :

$$
E=E_{0} \cdot e^{-0.002107 \cdot 2.84 \cdot t^{2} / 2}
$$

where $E_{0}$ is the initial Young's modulus of the cancellous bone and $t$ is the time in years. Through this law, authors conclude that under a 30-year pathology trabecular bone stiffness is reduced to about $90 \%$ of its initial value [36]. The new stiffness value for trabecular bone was implemented in the analysis, yielding the fracture paths shown in Fig. 6(b). Cortical bone stiffness has been also reduced to $90 \%$ of its initial value in order to include the effect of osteporosis. In addition, a simulation combining both osteoporosis has been included in this section, reducing both cortical and trabecular bone mechanical properties. The simulation of weakened trabecular bone representing osteoporosis leads to an increased fractured region as shown in Fig. 6(b). When cortical bone stiffness is reduced (Fig. 6(c)) due to the osteoporosis, the fracture path seems to be more localized, leading to only one simple crack path. When both osteoporosis are combined, an increased fracture zone appears in the central zone of the femoral neck, Fig. 6(d).

Concerning to falling conditions, Fig. 6(a) shows a long fracture path growing close to femoral head on the bottom zone. A second fracture appears in the lateral side of femoral 


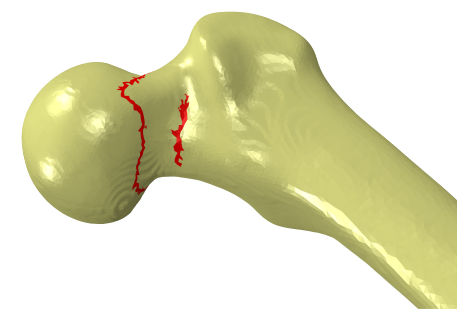

(a) Fracture under sideways falling condition

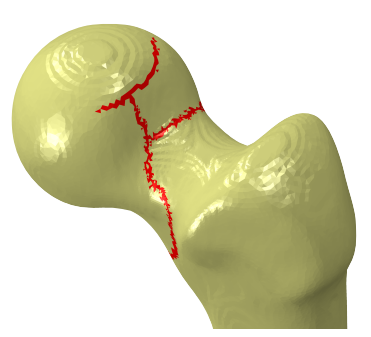

(b) Fracture with osteoporotic trabecular bone

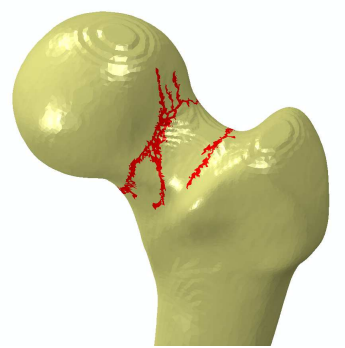

(d) Fracture with osteoporotic cortical and trabecular bone

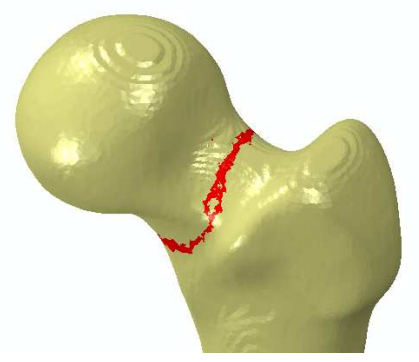

(c) Fracture with osteoporotic cortical bone

Figure 6: Femur fracture obtained under different conditions.

neck that tends to arrest. To the best of our knowledge, no published work simulating fracture under sideways fall conditions has been found, only elastic behaviour [8] or fracture load [37] have been analysed in the literature. When trabecular bone is affected by osteoporosis (Fig. 6(b)) the specimen shows a fracture path across the centre zone of femoral neck. In this case, the crack in the femoral head arrests and the final fracture is closer to the trochanteric area. However, when cortical bone is affected, Fig. 6(c), fracture appears in the central zone of the femoral neck, leading to an extracapsular fracture.

\subsection{Application to human femur fracture modelling}

Once the different techniques have been compared and applied to other configurations, the most reliable and efficient technique was also applied to a real human femur, taking into account a fracture criterion accounting for the heterogeneous nature of the bone. In this 
section, a human fresh-frozen cadaveric femur coming from an anonymous male donor was analysed. The specimen was provided by the University Complutense of Madrid, from the Centre of Body Donation and Dissection Areas, following the Spanish legislation. The donor had no reported history of muscle-skeletal diseases. Its anthropometric data are: right side, 73 years old, and donor with $170 \mathrm{~cm}$ height and $88 \mathrm{~kg}$ weight.

The experimental methodology was the same applied to synthetic bone, thus the femur was loaded in stance loading condition, increasing the load until fracture occurrence. Four different values of load were applied on the femoral head (500 N, 1000 N, $1500 \mathrm{~N}$ and 2000 N). The specimen was attached to the experimental rig as it is shown in Fig. 7(a). The femur was CT-scanned with a resolution of $0.2 \times 0.2 \times 0.2 \mathrm{~mm}^{3}$ and segmented using software ScanIP, obtaining a local distribution of the mechanical properties, relating them to the HU (Hounsfield Units) of the specimen. The FE mesh (shown in Figure 6b) was similar to that developed for the synthetic femur, with a refined mesh in the femoral neck in order to predict an accurate fracture path. The numerical model of the human femur was validated in the linear elastic range through several strain gauges and rosettes adhered to its surface. A comparison between experimental and numerical strains is shown in Fig. 7(c), showing a very good agreement between the experimental strain measures and the FE strain estimations. Concerning the fracture load of the human femur, a maximum value equal to $7120 \mathrm{~N}$ was obtained from the experimental test, while the numerical model estimated a value equal to $8178 \mathrm{~N}$, with a relative error of $15 \%$.

Due to the heterogeneity of the human femur, in this simulation mechanical properties are related to HU obtained in the scanner. Through the following relationships we consider the point-to-point heterogeneity of the bone, although we cannot take into account the non-isotropic behaviour (we note in passing that the degree of non-isotropy can vary largely from zone to zone). Linear elastic behaviour was assumed since it has been proved that linear FE models can properly predict the mechanical behaviour of the proximal femur [16]. Material properties were assigned to each element accounting for the level of HU from 


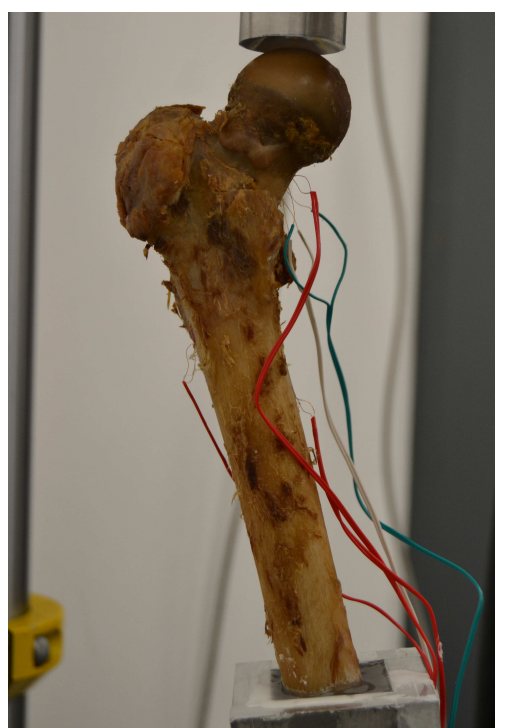

(a) Specimen positioned in the rig

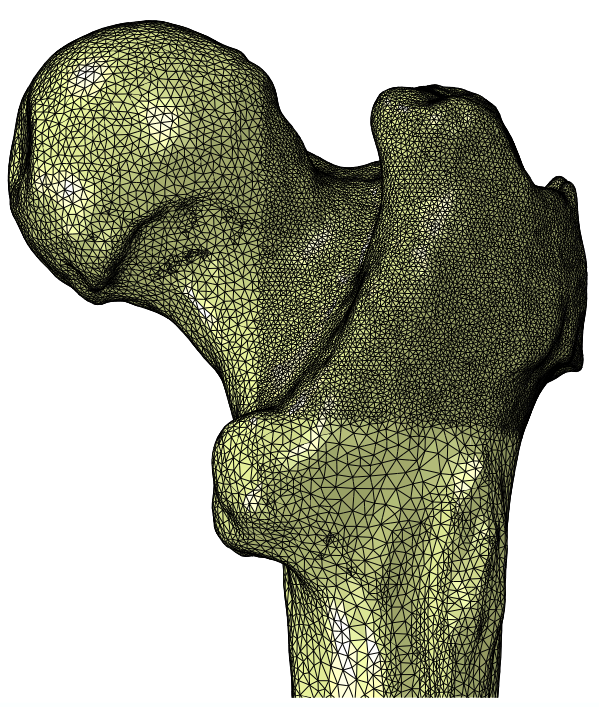

(b) Mesh developed using software ScanIP with a refined area in the femoral neck

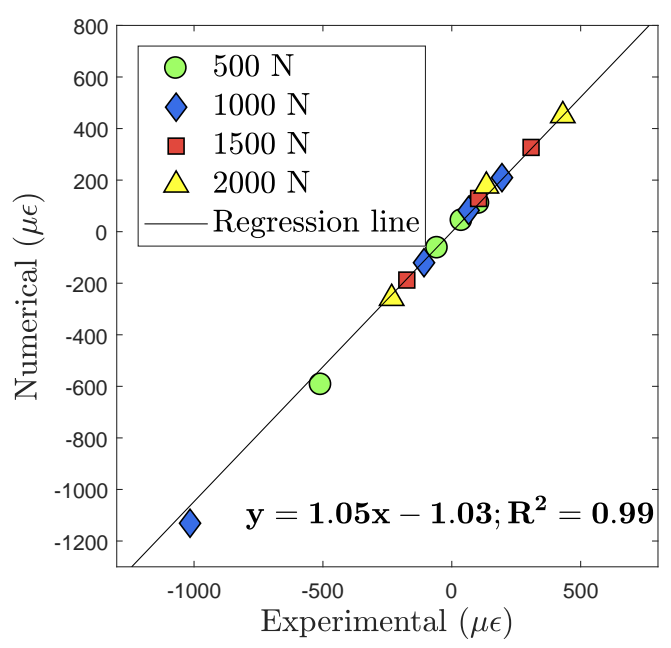

(c) Stiffness validation: comparison between experimental and numerical strains

Figure 7: Experimental test in human femur, mesh used in the numerical model and validation of stiffness. 
CT-scans: relationships between HU and proposed in [38] were implemented (Eqs. 6-8). Young's modulus was obtained from a density-based power law regression for the femoral neck [39] (Eq. 9).

$$
\begin{gathered}
\rho_{\mathrm{QCT}}\left(\mathrm{g} / \mathrm{cm}^{3}\right)=0.007764 \cdot H U-0.056148 \\
\rho_{\text {ash }}\left(\mathrm{g} / \mathrm{cm}^{3}\right)=0.877 \cdot \rho_{\mathrm{QCT}}+0.0789 \\
\rho_{\text {app }}\left(\mathrm{g} / \mathrm{cm}^{3}\right)=\rho_{\text {ash }} / 0.6 \\
E(\mathrm{MPa})=6850 \cdot \rho_{\text {app }}^{1.49}
\end{gathered}
$$

Crack propagation is modelled using the incremental crack growth technique explained above. Concerning the fracture criterion, a critical stress criterion has been used, using the relationships between $\sigma_{\text {crit }}$ and $\rho_{\text {app }}$ proposed by [40]. Relationships for femoral head and greater trochanter were stated as $\sigma_{\text {crit,head }}=22.6 \rho_{\text {app }}^{1.26}$ and $\sigma_{\text {crit,troc }}=50.1 \rho_{\text {app }}^{2.04}$ [40], respectively. Using these expressions, different $\sigma_{\text {crit }}$ for each material were calculated. Maximum principal stress at each element was compared to critical stress of the material through a USDFLD subroutine by the expression:

$$
f=\frac{\sigma_{\text {crit }}}{\sigma_{\text {max,ppal }}}
$$

Finally, elements with maximum values of $f$ were considered as failed elements and their mechanical properties were degraded (in terms of Young's modulus, reducing them to a very low value, $E=1 \mathrm{MPa}$ ), thus modelling the fracture evolution. In this specimen, fracture paths appeared along intertrochanteric line, as shown in Fig. 8, producing an extracapsular fracture

Fig. 8 shows accurate results when comparing experimental fracture and predicted fracture path, both internally in the femur and externally over the surface. Again, the numerical technique proposed in this work allows simulating long fracture paths also in real femurs. 


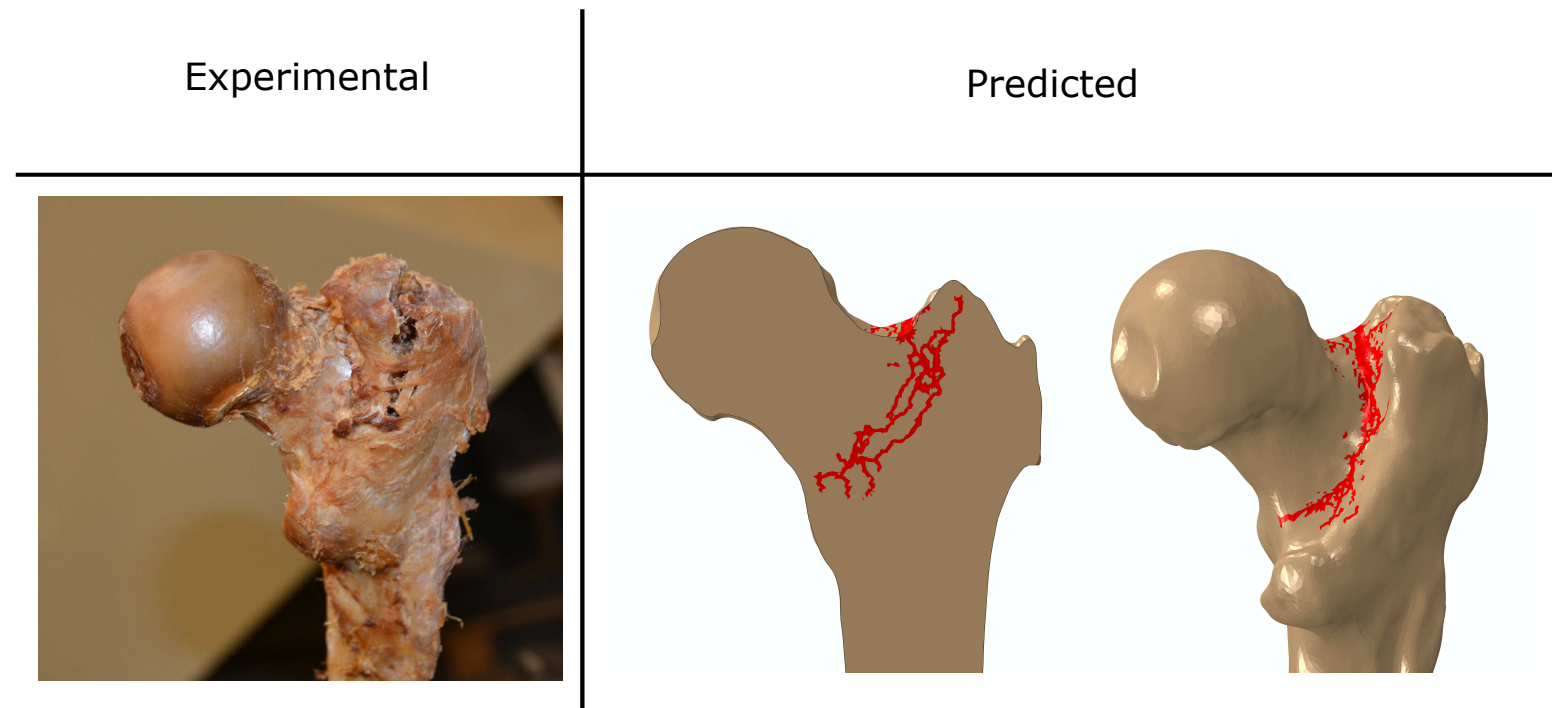

Figure 8: Comparison in fracture morphology obtained in experimental test and numerical model with human femur.

\section{Conclusions}

In this work, several techniques for numerical modelling of femur fracture propagation have been analysed. A FE model allowed the simulation of fracture evolution that is often poorly treated in the literature. Several subroutines have been tested, and also an incremental crack growth analysis has been developed through Python scripts. It has been concluded that the technique based on property degradation through incremental crack growth leads to the best results and performance in terms of convergence for this type of simulations. Using this technique, a realistic long crack path pattern has been obtained without convergence problems. Fracture pattern matched closely to experimental results, showing an intracapsular fracture as in the test of a synthetic femur. Other techniques, such as XFEM as implemented in Abaqus, do not lead to long fracture paths due to convergence problems.

Other loading and bone conditions were analysed with the same model: a sideways fall condition and an osteoporotic femur with reduced mechanical properties. The first shows a fracture closer to the femoral head and the latter a large crack pattern close to inter- 
trochanteric area. Finally, it has been proved that the selected technique together with a proper criterion is able to predict the fracture propagation in a real human femur, resulting in an extracapsular fracture. Through this technique, it is possible to simulate long fracture paths, which is important when fracture morphology is studied, since different fracture morphologies must be treated with distinct surgical treatments.

\section{Acknowledgements}

The authors are indebted to the Universidad Complutense de Madrid and Hospital Universitario Infanta Leonor for the support in the scanning and the testing human femur. The authors gratefully acknowledge the funding support received from the Spanish Ministry of Economy and Competitiveness and the FEDER operation program for funding the projects DPI2017-89197-C2-2-R, RTC-2015-3887-8 and the Generalitat Valenciana through the project Prometeo 2016/007.

\section{References}

[1] P. Carpintero, J.R. Caeiro, R. Carpintero, A. Morales, S. Silva, and M. Mesa. Complications of hip fractures: A review. World J. Orthop., 5(4):402-411, 2014.

[2] O. Johnell and J.A. Kanis. An estimate of the worldwide prevalence and disability associated with osteoporotic fractures. Osteoporosis Int., 17(12):1726-1733, 2006.

[3] M.J. Hall, C.J. DeFrances, S.N. Williams, A. Golosinskiy, and A. Schwartzman. National hospital discharge survey: 2007 summary. Natl. Health Stat. Report, 29(29):1-20, 2010.

[4] C.A. Brauer, M. Coca-Perraillon, D.M. Cutler, and A.B. Rosen. Incidence and mortality of hip fractures in the United States. Jama, 302(14):1573-1579, 2009.

[5] S.R. Cummings and L.J. Melton. Epidemiology and outcomes of osteoporotic fractures. The Lancet, 359(9319):1761-1767, 2002.

[6] E Michael Lewiecki. Osteoporosis. Ann. Intern. Med., 155(1):ITC1-1, 2011.

[7] M.M. Juszczyk, L. Cristofolini, and M. Viceconti. The human proximal femur behaves linearly elastic up to failure under physiological loading conditions. J. Biomech., 44(12):2259-66, aug 2011. 
[8] L. Grassi, E. Schileo, F. Taddei, L. Zani, M.M. Juszczyk, L. Cristofolini, and M. Viceconti. Accuracy of finite element predictions in sideways load configurations for the proximal human femur. J. Biomech., 45(2):394-399, 2012.

[9] Lorenzo Grassi, Sami P Väänänen, Saber Amin Yavari, Jukka S Jurvelin, Harrie Weinans, Matti Ristinmaa, Amir A Zadpoor, and Hanna Isaksson. Full-field strain measurement during mechanical testing of the human femur at physiologically relevant strain rates. J. Biomech. Eng., 136(11):111010, 2014.

[10] L. Cristofolini, M. Juszczyk, S. Martelli, F. Taddei, and M. Viceconti. In vitro replication of spontaneous fractures of the proximal human femur. J. Biomech., 40(13):2837-45, jan 2007.

[11] M. Doblaré, J.M. Garcıa, and M.J. Gómez. Modelling bone tissue fracture and healing: a review. Eng. Fract. Mech., 71(13):1809-1840, 2004.

[12] E. Giner, C. Arango, A. Vercher, and F.J. Fuenmayor. Numerical modelling of the mechanical behaviour of an osteon with microcracks. J. Mech. Behav. Biomed. Mater., 37:109-124, 2014.

[13] M. Marco, M. Rodríguez-Millán, C. Santiuste, E. Giner, and M.H. Miguélez. A review on recent advances in numerical modelling of bone cutting. J. Mech. Behav. Biomed. Mater., 44:179-201, 2015.

[14] M. Marco, E. Giner, R. Larraínzar-Garijo, J.R. Caeiro, and M.H. Miguélez. Numerical modelling of femur fracture and experimental validation using bone simulant. Ann. Biomed. Eng., 45(10):1-14, 2017.

[15] A. Ural and S. Mischinski. Multiscale modeling of bone fracture using cohesive finite elements. Engineering Fracture Mechanics, 103:141-152, 2013.

[16] E. Schileo, L. Balistreri, L. Grassi, L. Cristofolini, and F. Taddei. To what extent can linear finite element models of human femora predict failure under stance and fall loading configurations? J. Biomech., 47(14):3531-3538, 2014.

[17] L. Cristofolini, M. Viceconti, A. Cappello, and A. Toni. Mechanical validation of whole bone composite femur models. J. Biomech., 29(4):525-535, 1996.

[18] M.P. Gardner, A.C.M. Chong, A.G. Pollock, and P.H. Wooley. Mechanical evaluation of large-size fourth-generation composite femur and tibia models. Ann. Biomed. Eng., 38(3):613-620, 2010.

[19] T. Basso, J. Klaksvik, U. Syversen, and O.A. Foss. A biomechanical comparison of composite femurs and cadaver femurs used in experiments on operated hip fractures. J. Biomech., 47(16):3898-3902, 2014.

[20] R. Zdero, M. Olsen, H. Bougherara, and E.H. Schemitsch. Cancellous bone screw purchase: a comparison of synthetic femurs, human femurs, and finite element analysis. Proceedings of the Ins. Mech. Eng., Part H: J. of Eng. Med., 222(8):1175-1183, 2008.

[21] F. ONeill, F. Condon, T. McGloughlin, B. Lenehan, C. Coffey, and M. Walsh. Validity of synthetic bone as a substitute for osteoporotic cadaveric femoral heads in mechanical testing. Bone and Joint 
Research, 1(4):50-55, 2012.

[22] B. Pal, S. Gupta, A.M.R. New, and M. Browne. Strain and micromotion in intact and resurfaced composite femurs: experimental and numerical investigations. J. Biomech., 43(10):1923-1930, 2010.

[23] H. Ebrahimi, M. Rabinovich, V. Vuleta, D. Zalcman, S. Shah, A. Dubov, K. Roy, F.S. Siddiqui, E.H. Schemitsch, H. Bougherara, et al. Biomechanical properties of an intact, injured, repaired, and healed femur: an experimental and computational study. J. Mech. Behav. Biomed. Mater., 16:121-135, 2012.

[24] C. Kanchanomai, V. Phiphobmongkol, and P. Muanjan. Fatigue failure of an orthopedic implant-a locking compression plate. Eng. Fail. Anal., 15(5):521-530, 2008.

[25] L. Cristofolini, E. Schileo, M. Juszczyk, F. Taddei, S. Martelli, and M. Viceconti. Mechanical testing of bones: the positive synergy of finite-element models and in vitro experiments. Phil. Trans. Series A, Math., Phys., Eng. Sci., 368(1920):2725-63, 2010.

[26] Lorenzo Grassi, Sami P Väänänen, S.A. Yavari, H. Weinans, J.S. Jurvelin, A.A. Zadpoor, and H. Isaksson. Experimental validation of finite element model for proximal composite femur using optical measurements. J. Mech. Behav. Biomed. Mater., 21:86-94, may 2013.

[27] A.A. Ali, L. Cristofolini, E. Schileo, H. Hu, F. Taddei, R.H. Kim, P.J. Rullkoetter, and P.J. Laz. Specimen-specific modeling of hip fracture pattern and repair. J. Biomech., 47(2):536-543, 2014.

[28] J.H. Keyak and Y. Falkinstein. Comparison of in situ and in vitro CT scan-based finite element model predictions of proximal femoral fracture load. Med. Eng. Phys., 25:781-787, 2003.

[29] R. Hambli and S. Allaoui. A robust 3D finite element simulation of human proximal femur progressive fracture under stance load with experimental validation. Ann. Biomed. Eng., 41:12:2515-2527, 2013.

[30] N. Mos, J. Dolbow, and T. Belytschko. A finite element method for crack growth without remeshing. Int. J. Numer. Meth. Eng., 46(1):131-150, 1999.

[31] R.B. Cook and P. Zioupos. The fracture toughness of cancellous bone. J. Biomech., 42(13):2054-2060, 2009.

[32] E.A. Zimmermann, M.E. Launey, H.D. Barth, and R.O. Ritchie. Mixed-mode fracture of human cortical bone. Biomaterials, 30(29):5877-5884, 2009.

[33] E. Giner, N. Sukumar, J.E. Tarancón, and F.J. Fuenmayor. An Abaqus implementation of the extended finite element method. Eng. Fract. Mech., 76(3):347-368, 2009.

[34] B.L. Riggs, L.J. Melton, R.A. Robb, J.J. Camp, E.J. Atkinson, J.M. Peterson, P.A. Rouleau, C.H. McCollough, M.L. Bouxsein, and S. Khosla. Population-based study of age and sex differences in bone volumetric density, size, geometry, and structure at different skeletal sites. J. Bone Miner. Res., 19(12):1945-1954, 2004.

[35] Valérie Bousson, Françoise Peyrin, Catherine Bergot, Marc Hausard, Alain Sautet, and Jean-Denis Laredo. Cortical bone in the human femoral neck: Three-dimensional appearance and porosity using 
synchrotron radiation. J. Bone Miner. Res., 19(5):794-801, 2004.

[36] V.A. Lubarda, E.E. Novitskaya, J. McKittrick, S.G. Bodde, and P-Y Chen. Elastic properties of cancellous bone in terms of elastic properties of its mineral and protein phases with application to their osteoporotic degradation. Mech. Mat., 44:139-150, 2012.

[37] P. Varga, J. Schwiedrzik, P.K. Zysset, L. Fliri-Hofmann, D. Widmer, B. Gueorguiev, M. Blauth, and M. Windolf. Nonlinear quasi-static finite element simulations predict in vitro strength of human proximal femora assessed in a dynamic sideways fall setup. J. Mech. Behav. Biomed. Mater., 57:116-127, 2016.

[38] E. Schileo, E. DallAra, F. Taddei, A. Malandrino, T. Schotkamp, M. Baleani, and M. Viceconti. An accurate estimation of bone density improves the accuracy of subject-specific finite element models. J. Biomech., 41(11):2483-2491, 2008.

[39] E.F. Morgan, H.H. Bayraktar, and T.M. Keaveny. Trabecular bone modulus-density relationships depend on anatomic site. J. Biomech., 36(7):897-904, 2003.

[40] E.F. Morgan and T.M. Keaveny. Dependence of yield strain of human trabecular bone on anatomic site. J. Biomech., 34(5):569-577, 2001. 